\title{
Lossless Compression Algorithm For Color Image Based on the SHIRCT
}

\author{
Xinwen $\mathrm{Bi}^{1, \text { a }}$, Meng $\mathrm{Xu}^{1, \mathrm{~b}^{*}}$, Wenyou $\mathrm{Hu}^{1, \mathrm{c}}$ and Xiaodan $\mathrm{Shi}^{1, \mathrm{~d}}$ \\ ${ }^{1}$ Institute of Information Technology and Media, Beihua University, Jilin City, China \\ a52600613@qq.com,, ${ }^{\text {b3066567@qq.com, }{ }^{\text {}} 499154286 @ q q . c o m,{ }^{d} 85095462 @ q q . c o m}$
}

Keywords: Robust Control; Legendre Polynomials; Sure border; Voltage Control Strategy

\begin{abstract}
This paper studies the wavelet transform domain in low-frequency generation embedded SHIRCT transform complete color image compression algorithm. The results show that this algorithm can be very good to complete image lossless compression, by 12 color international standard test image simulation, JP2, RAR, ZIP ratio, PNG, TGA, PCX, TIF lossless compression results the average increase of: $-1 \%, 12 \%, 60 \%, 60 \%, 32 \%, 50 \%, 28 \%$.
\end{abstract}

\section{Introduction}

20 years, image compression technology has made great progress, the most representative of the wavelet transform theory ${ }^{[1]}$. Wavelet transform theory can be used to decompose the image signal into many subband signals ${ }^{[1]}$ with different spatial resolution, frequency characteristic and direction characteristic, and realize the simultaneous processing of low frequency and long time characteristics and high frequency short time characteristics ${ }^{[2]}$ The image signal is closer to the requirements of visual perception and data compression. Based on the wavelet transform, these features are widely regarded in the image compression coding technology, and various image compression schemes based on wavelet transform ${ }^{[3]}$ are presented. Among them, the SPIHT multi-level tree set splitting algorithm

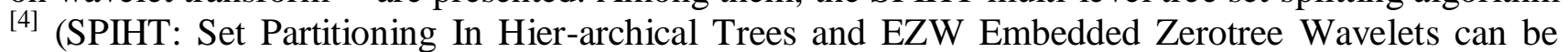
considered to represent the highest level of current zerotree wavelet coding ${ }^{[5]}$. In addition to selecting the effective wavelet compression algorithm, there is a need to consider the selection of wavelet bases. Different wavelet bases have different characteristics ${ }^{[6]}$. Different wavelet bases are used in the same compression algorithm to produce different compression effects. In this paper, the compression algorithm is improved based on the characteristics of wavelet compression combined with SHIRCT algorithm.

\section{SPIHT Algorithms}

SPIHT Algorithm Specific Symbols. $O(i, j)$ indicates the node ${ }^{(i, j)}$ a collection of all child coordinates.

$$
O(i, j)=\{(2 i, 2 j),(2 i, 2 j+1),(2 i+1,2 j),(2 i+1,2 j+1)\} .
$$

$D(i, j)$ represents the set of all the descendant coordinates of node $(i, j)$.

$\mathrm{H}$ denotes the set of transform coefficients of the largest scale of wavelet transform, both $\mathrm{LL}_{\mathrm{J}}$, $\mathrm{HL}_{\mathrm{J}}, \mathrm{LH}_{\mathrm{J}}$ and $\mathrm{HH}_{\mathrm{J}}$.

$$
L(i, j) \text { means } L(i, j)=D(i, j)-O(i, j) \text {. }
$$

Three lists:

(LIS), important pixel list (LIP), important pixel list (LSP), in LSP,LIP, ${ }^{(i, j)}$ represents a single pixel, in LIS, $(i, j)$ represents set $L(i, j)$ or $D(i, j)$. In order to distinguish between the two types of collections, if it is $D(i, j)$ said LIS table value for the A type, if it is $L(i, j)$ said LIS table value for the B type.

SPIHT Specific Implementation Process. I Initialization: 
$\underset{\text { Output }}{\mathrm{n}}=\left\lfloor\log _{2}\left(\max (\mathrm{i}, \mathrm{j})\left\{\left|\mathrm{C}_{\mathrm{i}}, \mathrm{j}\right|\right\}\right\rfloor\right.$, set the LSP is empty, the coordinate $(i, j) \in \mathrm{H}$ into the LIP, and $\mathrm{H}$ in the descendants (high frequency part: HLJ,LHJ,HHJ) into the LIS, as the A value.

II Sorting process:

(1) for each $(i, j) \in \mathrm{LIP}$

1) Output $S_{n}(i, j)$;

2) If $S_{n}(i, j)=1$, move $S_{n}(i, j)=1$ into the LSP and output the sign of $c_{(i, j)}$;

(2) for each $(i, j) \in$ LIS

1) If the value of $A$, then

(1) output $S_{n}(D(i, j))$;

(2) if $S_{n}(D(i, j))=1$, then for each $(k, l) \in O(i, j)$, as:

- Output $S_{n}(k, l)$;

- If $S_{n}(k, l)=1$, send $(\mathrm{k}, \mathrm{l})$ to the LSP and output its symbol;

- If $S_{n}(k, l)=0$, send $(\mathrm{k}, \mathrm{l})$ to the end of the LIP;

(3) If $L(i, j){ }_{\neq} \phi$, move $(\mathrm{k}, \mathrm{l})$ to the end of the LIS as the B value; otherwise, remove $(\mathrm{i}, \mathrm{j})$ from the LIS.

2) If the value of $B$, then

(1) output $S_{n}(L(i, j))$;

(2) if $S_{n}(L(i, j))=1$, then

- for each $(k, l) \in O(i, j)$ added to the end of the LIS as an A value;

- Delete $(i, j)$ from LIS.

(3) refinement process: for each $(i, j) \in L S P$ (not including the last splitting process), output the nth most significant bit of $\left|c_{i, j}\right|$;

(4) quantization step size refresh: $n=n-1$; return (2).

\section{SHIRCT Transformations}

The SHIRCT transform involves three bands of transformations. One advantage of the SHIRCT transform is that the transform can be done entirely by addition and shift, and the operation is fast and hardware is complete.

Positive Transform

$\mathrm{t}=\mathrm{X} 1-((\mathrm{X} 2+\mathrm{X} 3))>>1)$.

$\mathrm{Z} 1=\mathrm{X} 2+\mathrm{X} 3+(\mathrm{t} \gg>1)$

$\mathrm{Z} 3=-\mathrm{X} 2+((\mathrm{Z} 1+(\mathrm{t}>>3))>>1)$;

$\mathrm{Z} 2=\mathrm{t}+\eta \mathrm{Z3}$

Inverse Transformation

$\mathrm{t}=\mathrm{Z} 2-\eta \mathrm{Z} 3$;

$\mathrm{X} 2=-\mathrm{Z} 3+((\mathrm{Z} 1+(\mathrm{t}>>3))>>1)$;

$\mathrm{X} 3=\mathrm{Z} 1-\mathrm{X} 2+(\mathrm{t} \gg>1)$

$\mathrm{X} 1=\mathrm{t}+((\mathrm{X} 2+\mathrm{X} 3)>>1)$

In the above transformation, the $\mathrm{Z} 2$ component can be expressed as:

$$
\begin{aligned}
& Z_{2}=\left(\frac{5}{16} X_{r}+\frac{21}{32} X_{2}+\frac{11}{32} X_{3}\right) \eta+ \\
& X_{1}-\frac{X_{2}-X_{3}}{2}=Z_{3} \eta+X_{1}-\frac{X_{2}+X_{3}}{2}
\end{aligned}
$$


Considering the actual effect of image compression, it is desirable that $|\mathrm{Z} 2|$ be as small as possible, since $\mathrm{Z} 2$ is a parameterized expression, and Z3, X1- $(\mathrm{X} 2+\mathrm{X} 3) / 2$ are both fixed values, so the choice of $\eta$ The effect is very large, different $\eta$ represents a different reversible transformation, theoretically such a reversible transformation has an infinite number, but always want to find the best one.

In order to facilitate the hardware implementation, the transformation is completely composed of addition and shift, $\eta$ can be written $\eta=\mathrm{k} / 21(\mathrm{k} \neq 0,1 \geq 0, \eta$ symbol given directly in the algorithm), rewrite the parameters in the transformation as follows:

Positive Transform

$$
\mathrm{Z}_{2}=\left(\mathrm{t} \pm \sum_{i=1}^{q} Z_{3}\right)>>1
$$

Inverse transformation

$$
\mathrm{t}= \pm\left(\sum_{i=1}^{q} Z_{3}\right)>>1
$$

Encoding Process

In this paper, the wavelet algorithm is adopted at the front end, then the SPIHT algorithm is applied to the wavelet coefficients, and then the new color image compression algorithm is obtained by SHIRCT transform. Decoding is the inverse of the encoding, including the three steps corresponding to the forward SPIHT: restore the quantization step refresh, restore the prediction and sort.

Experimental Results and Conclusions

In order to illustrate the effectiveness of the algorithm, this paper compares the lossless image compression algorithms of JP2, RAR, ZIP, PNG, TGA, PCX and TIF in 12 color international standard test images, and the average compression ratio is not Than the above algorithm were increased by $-1 \%, 12 \%, 60 \%, 60 \%, 32 \%, 50 \%, 28 \%$, see Table 1 .

Table 112 color international standard test image compression experiment comparison results

\begin{tabular}{|c|c|c|c|c|c|c|c|}
\hline $\begin{array}{c}\text { Compress } \\
\text { ion } \\
\text { scheme } \\
\begin{array}{c}\text { InternaTi } \\
\text { onal } \\
\text { Standard } \\
\text { Test } \\
\text { Iimages }\end{array}\end{array}$ & JP2 & RAR & PCX & TGA & ZIP & TIF & PNG \\
\hline Kodak1 & 511631 & 598789 & 1161410 & 1157891 & 796111 & 1065614 & 783060 \\
\hline Kodak2 & 451678 & 508771 & 1142233 & 1150300 & 665774 & 865916 & 621478 \\
\hline Kodak3 & 399015 & 458212 & 1096862 & 1085783 & 569796 & 890712 & 549676 \\
\hline Kodak4 & 461292 & 512279 & 1172752 & 1153733 & 747996 & 108740 & 640877 \\
\hline Kodak5 & 533178 & 684773 & 1185005 & 1162343 & 915984 & 1238244 & 810363 \\
\hline Kodak6 & 472736 & 532397 & 1188493 & 1148689 & 718077 & 1001630 & 673733 \\
\hline Kodak7 & 419224 & 488048 & 1113169 & 1109121 & 650774 & 976216 & 573980 \\
\hline Kodak8 & 548967 & 804423 & 1309333 & 1163564 & 951692 & 1255784 & 791697 \\
\hline Kodak9 & 446250 & 488367 & 1190110 & 1152950 & 619045 & 838012 & 587850 \\
\hline Kodak10 & 454440 & 495213 & 1171219 & 1154093 & 686399 & 958320 & 598508 \\
\hline Kodak11 & 458044 & 553611 & 1139853 & 1129959 & 699897 & 930316 & 643047 \\
\hline Kodak12 & 426781 & 482724 & 1247133 & 1130272 & 598262 & 881932 & 575313 \\
\hline $\begin{array}{c}\text { SPIHT+ } \\
\text { MRCT }\end{array}$ & $2 \%$ & $15 \%$ & $51 \%$ & $51 \%$ & $34 \%$ & $52 \%$ & $31 \%$ \\
\hline
\end{tabular}




\section{Acknowledgements}

This work has been the Ministry of Education "Chunhui plan" (104900071,1049000150),Jilin Province Science and Technology Development Project Project Research Achievements (20170418035FG), Beihua University Education and Teaching Reform Project: Development of Immersive (VR) Experimental Platform for Innovative Talents Training and Resource Construction; Research on the Design and Teaching Application of Micro - course for User Experience Financial support for the reform project of the North China University (XJQN2016035, XJYB2016025).

\section{References}

[1] Shapiro J M. Em bedded image coding using zerotrees of wavelet coefficients [ J ] . IEEE Transactions on Signa 1 Processing, 1993, 41 ( 12) : 3445 - 3462.

[2] Said A, Pearlman W. A new fast and efficient image codec based on set partitioning in hierarchical trees [ J ] . IEEE Transactions on Circuits System Video Technology, 1996, 6( 6 ): $243-250$.

[3] Geronimo J S, Hard in D P. Fractal functions and wavelet expansions based on several scaling function s [ J ]. Journal of Approx Theory, 1994, 78( 3 ): 373 - 401.

[4] Khan E, GhanbariM. Very low bit rate video coding us ingvirtual SPIHT [ J]. IEEE E lectron ics Let ters, 2001, 37( 1 ): 40 - 42.

[5] ZhengWu, Yu Sheng-shen, Zhou Jing- 1,ietal. Orthogonal balanced multi wavelet and its study on image coding $[\mathrm{J}]$. Journal of Computer A ided Design \& Computer Graphics, 2004, 16 ( 11 ): $1490-1495$.

[6] Zheng Wu, Yu Shengsheng, Zhou Jingli et al. Construction of orthogonal equalization multiwavelets and its research in image coding [J] .Journal of Computer Aided Design and Graphics, 2004, 16 (11): 1490 - 1495. ]

[7] Martin M B, Bell Amy E. New image compression techniques using multiwavelets and multiw avelet packets [ J ] . IEEE T ransactions on Image Processing, 2001, 10( 4 ): 500 - 510. 